\title{
Dislocation Emission around Nanoindentations on a (001) fcc Metal Surface Studied by STM and Atomistic Simulations
}

\author{
${ }^{1}$ O. Rodríguez de la Fuente日, ${ }^{2}$ J. A. Zimmerman, ${ }^{1}$ M. A. González, ${ }^{2}$ J. de la Figuera, ${ }^{2}$ J. C. \\ Hamilton, ${ }^{3}$ Woei Wu Pai, ${ }^{1}$ J. M. Rojo \\ ${ }^{1}$ Departamento de Física de Materiales, Universidad Complutense, Madrid 28040, Spain \\ ${ }^{2}$ Sandia National Laboratories, Livermore, California 94550, USA \\ ${ }^{3}$ Center for Condensed Matter Sciences, National Taiwan University, Taiwan 106
}

(November 2, 2018)

\begin{abstract}
We present a combined study by Scanning Tunneling Microscopy and atomistic simulations of the emission of dissociated dislocation loops by nanoindentation on a (001) fcc surface. The latter consist of two stacking-fault ribbons bounded by Shockley partials and a stair-rod dislocation. These dissociated loops, which intersect the surface, are shown to originate from loops of interstitial character emitted along the $<110>$ directions and are usually located at hundreds of angstroms away from the indentation point. Simulations reproduce the nucleation and glide of these dislocation loops.
\end{abstract}

PACS numbers: 68.55.Ln, 68.35.Gy, 61.72.Bb, 61.72.Ff

Typeset using REVTEX 
Nanoindentation is a well-recognized technique that elucidates mechanical properties of surfaces and thin films at atomic scale [1 3]. Although it is the small-scale counterpart of the traditional hardness test, it is far from being understood at the same level. Experimental evidence is still rather fragmentary although a number of studies involving atomic force microscopy and related techniques have provided information about such remarkable phenomena as the passivating properties of self-assembled molecular single layers [4] or the softening role of steps in the onset of plasticity [5]. Although most of our initial knowledge of the processes taking place under a nanoindenter comes from molecular dynamics simulations, in which much progress has been made in recent years [6,7], a limiting factor of the comparison of atomistic simulations and experiment has been the different length scales accessible to each. In the present work we have succeeded to bridge the length-scale gap by increasing the experimental resolution around the nanoindentation point down to atomic scale.

The nucleation of discrete dislocations and their interactions are crucial to understand the first steps of crystal plasticity. In the present Letter, we report the first direct observation by Scanning Tunneling Microscopy (STM) of the emission of individual dislocation loops by nanoindentation of a $\mathrm{Au}(001)$ surface and show that a combined STM and atomistic simulation investigation explains how these loops are originated and how they glide away hundreds of angstroms from the indentation point. We fully characterize these loops and present evidence of the atomic displacement processes that are responsible for their generation. Furthermore, standard dislocation theory is shown to provide a suitable and convenient framework for encompassing the experimental results and the atomic simulations.

The experiments were performed in ultra high vacuum conditions on a $\mathrm{Au}(001)$ crystal cleaned by repeated sputtering and annealing in a system described in more detail elsewhere [8]. Nanoindentations were done pushing the tungsten tip against the sample for distances of a few nanometers from the stable tunnel distance with the control feedback switched off. The last layer of the $\mathrm{Au}(001)$ surface is reconstructed with the well known "hex" $5 \times 20$ reconstruction which appears in STM images as fringes oriented along a $<110>$ direction. 
These fringes arise from a moiré-like effect between the topmost layer with an hexagonal arrangement and the lower bulk-like layer with square symmetry.

A typical image of the surface of the crystal after performing nanoindentations is shown in Fig. 1. Indentations themselves are imaged as multi-storied pits, and some material is shown as a pile-up surrounding the pits. Rows of bump-like features with a height of 0.6 $\pm 0.1 \AA$ (called from now on hillocks) are apparent along the compact $<110>$ directions on the (001) surface, at distances of hundreds of angstroms from the indentation points. We have also observed similar hillocks in the course of ion irradiation on both $\mathrm{Au}(001)$ and $\mathrm{Ag}(001)$ followed by gentle annealing: in these cases the hillock distribution is random, with no alignments in rows as the ones surrounding nanoindentations.

Close-up STM images of hillocks are shown in Fig. 2 together with an interpretation of their sub-surface structure [8], which consists of two stacking-faults on intersecting $\{111\}$ planes each bounded by two parallel Shockley partial dislocations. The whole configuration is held up by a stair-rod dislocation parallel to the surface. We argue that the origin of the hillocks can be traced to perfect dislocation loops that are punched into the crystal by the tip displacement into the surface [9]. In our case, a family of such dislocation loops would consist of $\mathrm{V}$-shaped half-loops intersecting the surface with Burgers vector parallel to the latter. They would, on energetic grounds, be split into two pairs of Shockley partials giving rise to the configuration shown in Fig.2b, i.e. resulting in hillocks [10]. We can ascertain that these loops are of interstitial character based on the fact that for $\mathrm{Au}$ (Fig. 2a) we observe a missing reconstruction fringe on top of the hillock. As the interatomic spacing of the substrate is larger than the one of the uppermost reconstructed layer, the position of the missing-fringe in the moiré-like pattern [11] corresponds to the position of the extra row of interstitial atoms below. Furthermore, for the $\mathrm{Ag}(001)$ atomic resolution image of Fig. 2 c, it is clear that each partial dislocation produces a mismatch of one half interatomic unit between the rows on both sides of each stacking-fault, the two mismatches adding up to one extra row of atoms in the inner side of the hillock.

To gain insight into the atomic processes involved in the creation of these hillocks, atom- 
istic simulations [12] were carried out. A repulsive potential [6] was used to model a spherical indenter of radius $4 \mathrm{~nm}$ penetrating the surface of a $\mathrm{Au}(001)$ crystal modeled with the embedded atom method potential [13]. The reconstructed layer is thought to behave like a floating layer [14]. Thus, it is not expected to affect dislocation generation and behavior during nanoindentation and was not included in the simulations. A top view of the surface after the simulated nanoindentation is shown in Fig. 3. In agreement with experiment, it is observed that two hillocks have been generated around the nanoindentation trace. To make a more quantitative comparison between simulations and dislocation models, a quantity closely related to the Burgers vector can be defined for every atom [15]. This quantity is called the slip-vector, $\mathbf{s}_{i}$, being defined for atom $i$ as:

$$
\mathbf{s}_{i}=-\frac{1}{N_{s}} \sum_{j \neq i}^{N_{n n}}\left(\mathbf{r}_{i j}-\mathbf{r}_{i j}^{0}\right)
$$

where $\mathbf{r}_{i j}$ and $\mathbf{r}_{i j}^{0}$ are the vectors linking atom $i$ and all its $N_{n n}$ nearest neighbors $j$ in their current and reference (prior to the indentation) positions, respectively. $N_{s}$ stands for the number of slipped neighbors. The spatial distribution of the slip vectors moduli $\left|\mathbf{s}_{i}\right|$ around the nanoindentation trace, with a suitable color scale, is shown in Fig. 1. Green atoms with $\left|\mathbf{s}_{i}\right| \sim \frac{a_{0}}{\sqrt{6}}$ are atoms on a stacking fault ( $a_{0}$ is the Au lattice parameter). Blue atoms correspond to values of the slip-vector modulus between the stacking fault value and zero, while yellow ones have a slip-vector ranging between a complete lattice parameter and the stacking fault value. Blue and yellow atoms are, consequently, around the core of a Shockley partial dislocation. The configuration shown in Fig. 1, with a height displacement for the topmost atoms of $0.6 \AA$, strikingly reproduces the previously proposed subsurface structure of a hillock.

A dynamical picture of the process is obtained by recording successive frames of the simulated atomic events. To obtain the sequence, the indenter is first lowered in increments of $0.01 \mathrm{~nm}$ down to a depth of $0.58 \mathrm{~nm}$ in a quasi-static way at zero temperature. At this point dislocation loops are observed below the indenter, in agreement with previous results [6]. Then, a constant energy molecular dynamics simulation follows the evolution of 
the system for 36 ps. A different dislocation configuration (again reproducing the previously proposed subsurface structure of a hillock) is created close to the tip and, then, glides away in a $<110>$ direction. We stress that, although the experimentally observed hillocks are usually much larger than the simulated ones (and the indentation itself is also much deeper), we observe also hillocks of the same size in both experiment and simulation.

Hillocks are seen to glide as a whole unit. This behavior can be understood on the grounds that Shockley partial dislocations bounding a stacking fault are expected to glide easily on the $\{111\}$ gliding planes. In our simulation they indeed glide away dragging with them the stair-rod dislocation (motion of structures formed by stair-rod dislocations and Shockley partial dislocations in thin films has been recently reported [16]). We argue that the rows of hillocks appearing in the STM image of Fig. 1 are the result of successive emission of loops that glide away from the nanoindentation trace. Once started into motion due to the high stress close to the indentation, the hillocks would glide away from the indentation point until they collide or interact with other defects in the crystal. The simulation cell is too small to observe in detail this effect, although the hillocks move with a constant velocity within the unit cell once they are far enough from the indentation point. Hillock-like structures (although interpreted in a different way) have been observed to drift in highly stressed regions [17]. The creation of hillocks following ion irradiation can also be explained in terms of the above model: after long ion irradiation and further annealing, the surface is known to exhibit a multi-storied pit structure [18], whereas the presence of a large supersaturation of adatoms and, probably, sub-surface interstitials, is suspected. These extra-atoms can cluster on crystallographic planes and, after relaxation, give rise to perfect loops that start the above mechanism.

In the course of the many simulations performed, a variety of hillock structures have been found. They differ in the exact arrangement of the loop below the surface: more complex configurations than the simplest one observed in Fig. 田 (an edge V-shaped loop dissociated along $\{111\}$ planes) are possible. But the defining characteristics are the same for all of them: the Burgers vector of the undissociated loop is a lattice vector, all the sections of the 
loop appear dissociated along $\{111\}$ planes, and the loop glides as a whole unit in a $<110>$ direction. It is also worth remarking that, in the simulations, the hillocks remain in place once the tip is retracted from the surface, in agreement with the experiment.

Comparing different experimental images, it is observed that the size distribution of both the span $s$ (distance between stacking faults at the surface), and width $w$ (separation between partial dislocations again at the surface) of the observed hillocks is rather broad, ranging from a lowest resolvable size of about one reconstruction period $(\sim 1.4 \mathrm{~nm})$ to a size of several ones. However, the parameter $w$ (in fact, the width of the extended dislocation) is experimentally seen to level off with increasing span $s$ of the hillock. The repulsive interaction force between two Shockley partial dislocation segments, at a given distance, increases initially with segment length (which is proportional to the observed span) but attains a constant value when this length becomes much larger than the distance between the segments. Within a model using elemental dislocation theory [19] and taking into account image dislocations to include the effect of the free surface, the exact form of the $w(s)$ curve can be predicted and is found to be in agreement with the experimental data [20]. It is worth emphasizing that the parameters (Burgers vectors, geometry...) of this new configuration can be explained in terms of continuum dislocation theory.

In summary, we have shown that the initial stages of plastic deformation around a nanoindentation result in the emission from near the contact point of dislocation half-loops intersecting the surface; they can, alternatively, be created by accretion of irradiated-in interstitials. These loops are split into pairs of Shockley partial dislocations giving rise to peculiar configurations at the surface (hillocks), involving four Shockley partial dislocations and a stair-rod which can be analyzed in terms of standard dislocation theory. Dissociated loops glide across compact planes and this provides a novel mechanism for matter transport away from nanoindentations. The observation of these defects in other systems, such as $\operatorname{Ag}(001)$, suggests that our results can be generalized to other (001) fcc metal surfaces, thus providing a consistent description with an unprecedented resolution of the incipient plastic deformation mechanisms. This might help the interpretation of elementary yielding events 
observed in previous nanoindentation investigations [3, 2, 5].

This research was supported by Spanish CICyT through Project No. PB96-0652 and by the Office of Basic Energy Sciences, Division of Materials Sciences, U.S. Department of Energy under contract No. DE-AC04-94AL85000. O.R.d.l.F gratefully acknowledges support from the Spanish MEC. 


\section{REFERENCES}

* Corresponding author. Fax: +34 9139445 47. E-mail: oscar@material.fis.ucm.es

[1] U. Landman, W. D. Luedtke, Nancy A. Burnham and Richard J. Colton, Science 248, 454 (1990).

[2] S. G. Corcoran, R. J. Colton, E. T. Lilleodden and W. W. Gerberich, Phys. Rev. B 55 16057 (1997).

[3] J. D. Kiely and J. E. Houston, Phys. Rev. B 5712588 (1998).

[4] Ross C. Thomas, J. E. Houston, Terry A. Michalske and Richard M. Crooks, Science 259, 1883 (1993).

[5] J. D. Kiely, R. Q. Hwang, and J. E. Houston, Phys. Rev. Lett. 81, 4424 (1998).

[6] C. L. Kelchner, S. J. Plimpton, and J. C. Hamilton, Phys. Rev. B 58, 11085 (1998).

[7] E. B. Tadmor, R. Miller, R. Phillips and M. Ortiz, J. Mat. Res. 14, 2233 (1999).

[8] J. de la Figuera, M. A. González, R. García-Martínez, J. M. Rojo, O. S. Hernán, A. L. Vázquez de Parga and R. Miranda, Phys. Rev. B 58, 1169 (1998).

[9] O. Rodríguez de la Fuente, M. A. González, and J. M. Rojo, in Fundamentals in Nanoindentation and Nanotribology II, edited by S. P. Baker et al. (MRS, Boston, 2001).

[10] In terms of the Thompson's tetrahedron notation, the perfect loop segment on the $\bar{a}$ plane would split in this plane under the reaction $\boldsymbol{C D} \rightarrow \boldsymbol{C} \boldsymbol{\alpha}+\boldsymbol{\alpha} \boldsymbol{D}$. The dissociation of both segments on the $\bar{a}$ and $\bar{b}$ planes, respectively, gives rise to the stacking faults intersecting at the stair-rod dislocation with Burgers vector $\boldsymbol{\alpha} \boldsymbol{\beta}$.

[11] P. B. Hirsch et al., Electron microscopy of thin crystals, pg. 375 (Butterworths, London, 1965).

[12] The cell dimensions are $20.4 \mathrm{~nm} \times 20.4 \mathrm{~nm} \times 12.5 \mathrm{~nm}$ with 300.000 atoms. The top 
surface is traction free, while the bottom is held fixed with periodic boundary conditions applied to the side surfaces.

[13] S. M. Foiles and M. I. Baskes, and M. S. Daw, Phys. Rev. B 33, 7983 (1986).

[14] V. Fiorentini, M. Methfessel and M. Scheffler, Phys. Rev. Lett. 71, 1051 (1993).

[15] Zimmerman, Kelchner, Klein, Hamilton and Foiles, Phys. Rev. Lett. in review.

[16] J. de la Figuera, K. Pohl, O. Rodríguez de la Fuente, A. K. Schmid, N. C. Bartelt, C. B. Carter and R. Q. Hwang Phys. Rev. Lett. 86, 3819 (2001).

[17] Z. Gai, Y. He, X. Li, J. F. Jia and W. S. Yang, Surf. Sci. 365, 96 (1996).

[18] O. Rodríguez de la Fuente, M. A. González and J. M. Rojo, Phys. Rev. B 63, 085420 (2001).

[19] J. P. Hirth and J. Lothe, Theory of dislocations, (Krieger, Malabar, FL, 1992).

[20] O. Rodríguez de la Fuente et al., unpublished. 


\section{FIGURES}

FIG. 1. $\left(98 \times 98 \mathrm{~nm}^{2}\right) \mathrm{STM}$ image of two nanoindentations in the $\mathrm{Au}(001)$ surface. Rows of hillocks stemming from the nanoindentation points and following a $<110>$ direction are visible. Bumps of pile-up material surround nanoindentation points (the contrast in these bumps is saturated to enhance the visibility of the hillocks). Capital letters on one of the hillocks are used to compare their orientations with the ones in Fig. 2 .

FIG. 2. a) $\left(25 \times 25 \mathrm{~nm}^{2}\right) \mathrm{STM}$ image in $\mathrm{Au}(001)$ of a hillock, such as seen near nanoindentation points. b) Scheme of the dislocation configuration proposed for dissociated loops. Burgers vectors in the Thompson tetrahedron notation and line directions are shown for each segment. c) $(11.6 \times 11.6$ $\left.\mathrm{nm}^{2}\right)$ A hillock in a $\mathrm{Ag}(001)$ surface previously ion-bombarded and annealed. Note the atomic resolution and the positions of the emerging partial dislocations.

FIG. 3. Top view of the nanoindentation simulation. The dark region in the middle of the picture corresponds to the indentation point. Two hillocks are emitted along $<110>$ directions. Note the striking similarity of these simulated defects with the experimental image of Fig. 2ac.

FIG. 4. (color) 3D simulation side views from different orientations of the dissociated dislocation loop (corresponding to the sub-surface configuration of Fig. 3), colored according to the slip vector. Atoms which signify a stacking-fault plane are colored green. Blue and yellow atoms define the core of the leading and the trailing partial dislocations, respectively. Red designs atoms which have slipped a full $<110>$ vector. Black arrows indicate the indentation point and its axis. The bottom picture can be obtained rotating the top one about $90^{\circ}$ counterclockwise around the nanoindentation axis. 


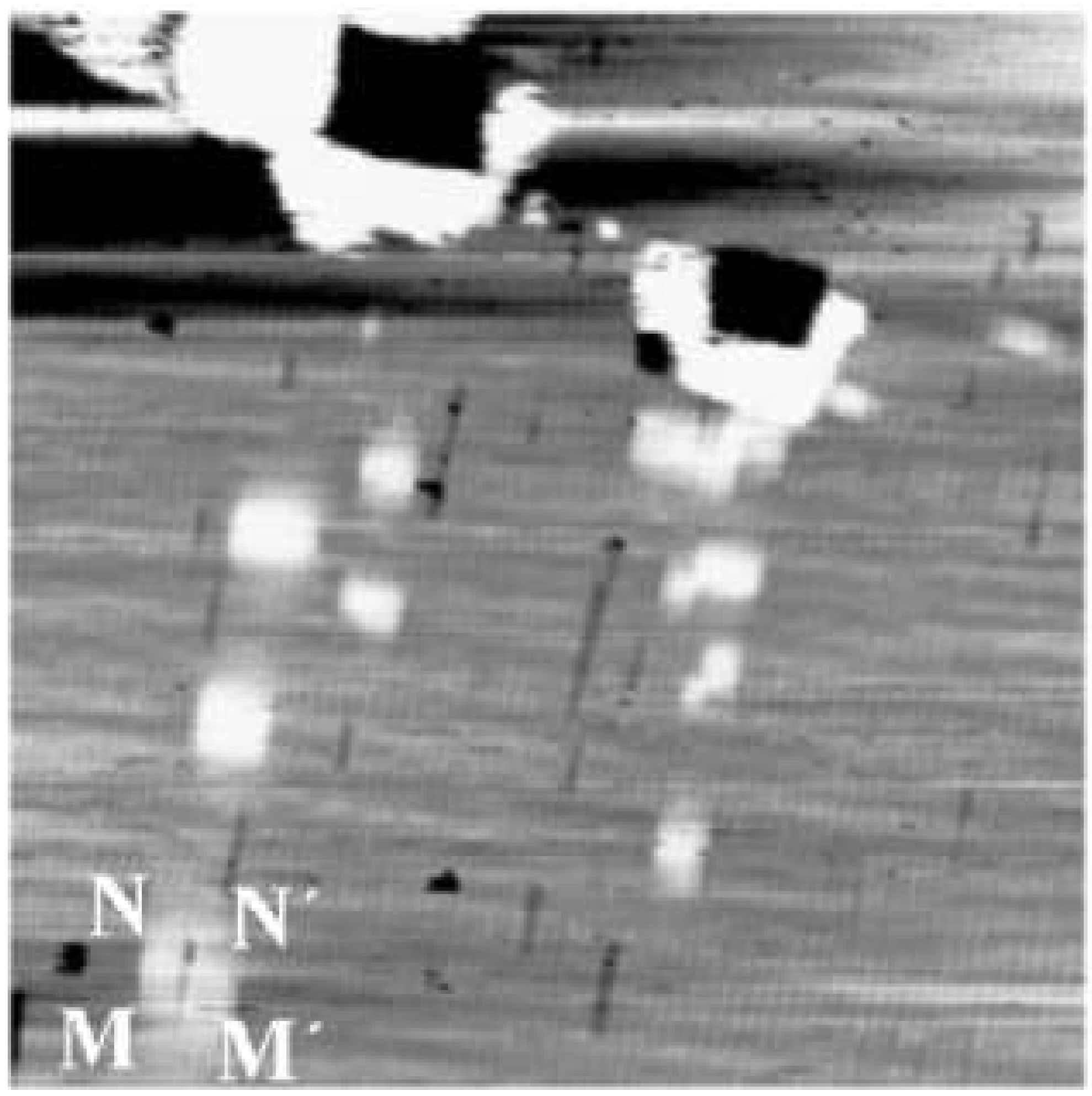



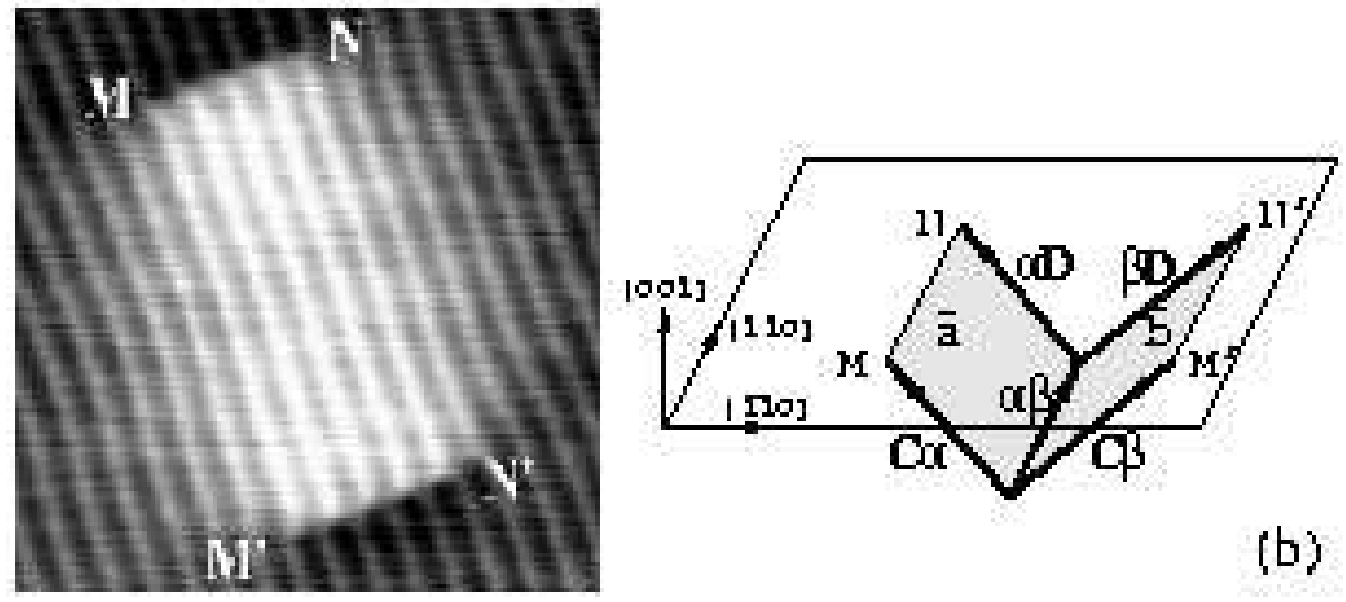

(b)

(a)

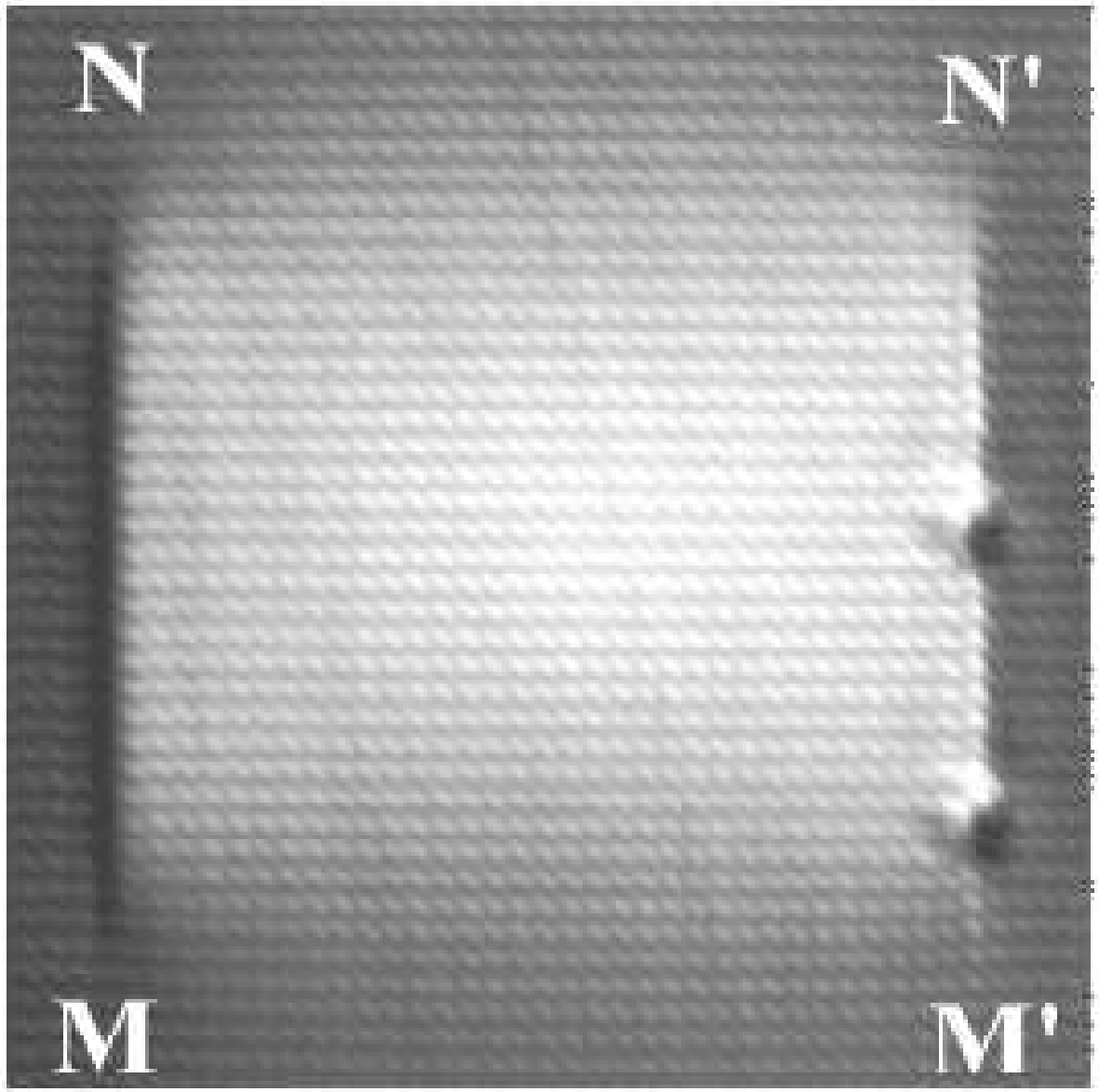

(c) 


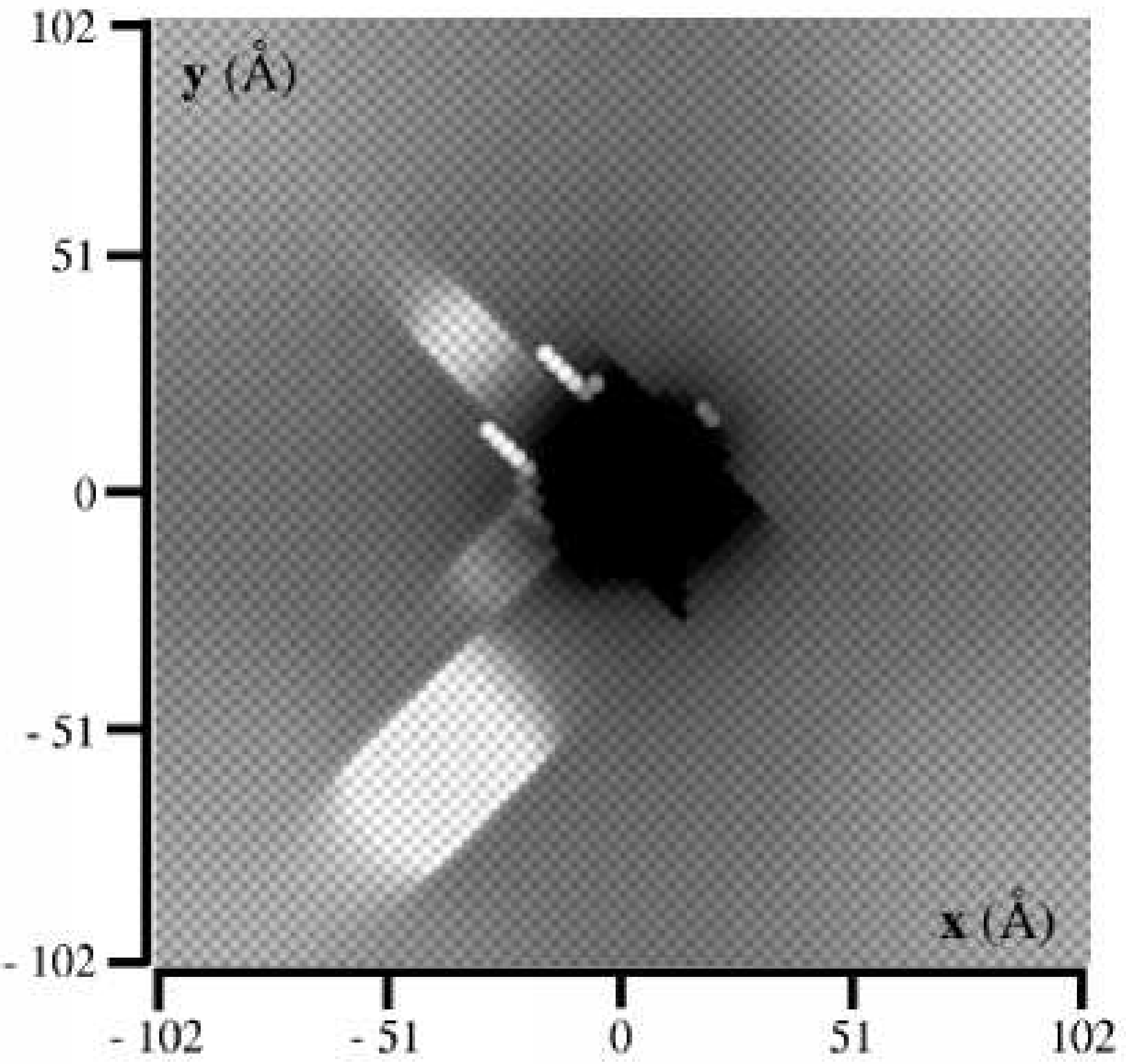




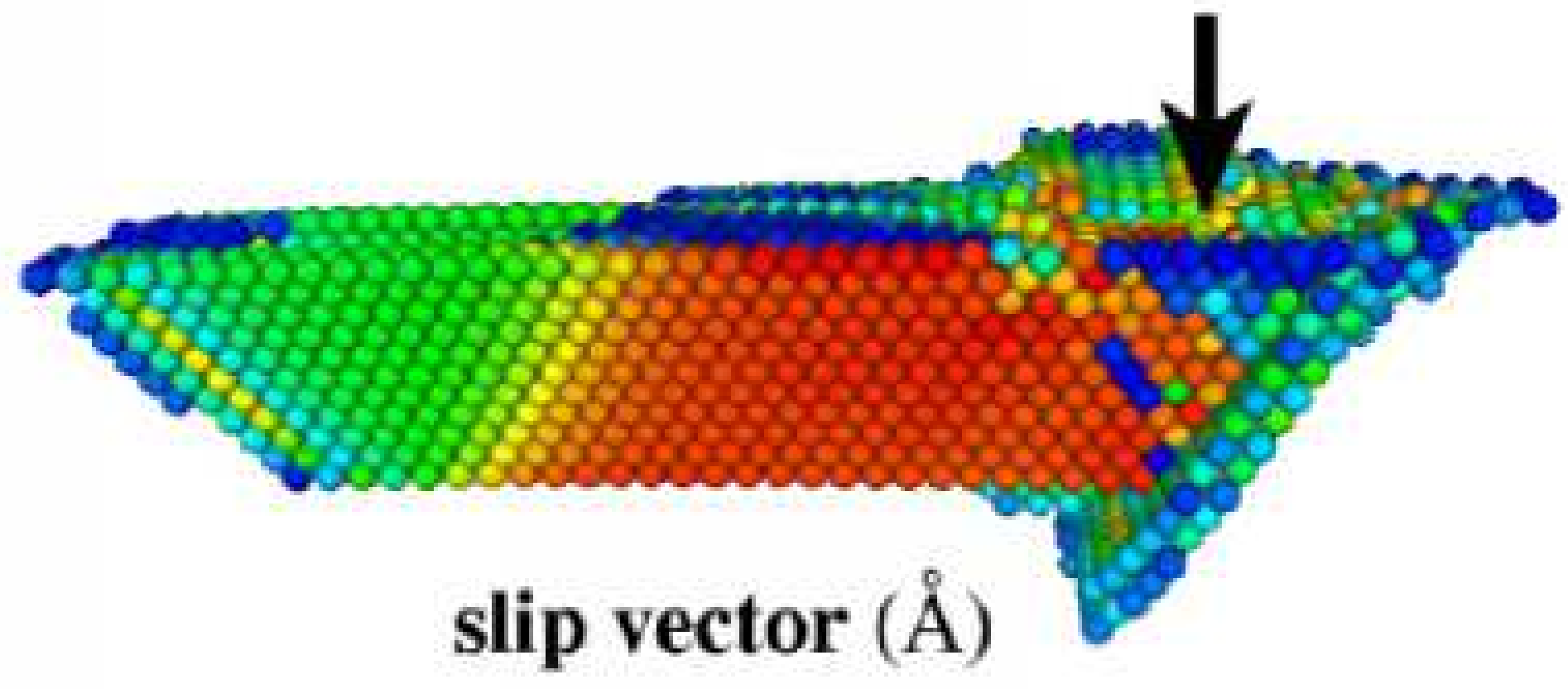
0.38
1.70
3.01
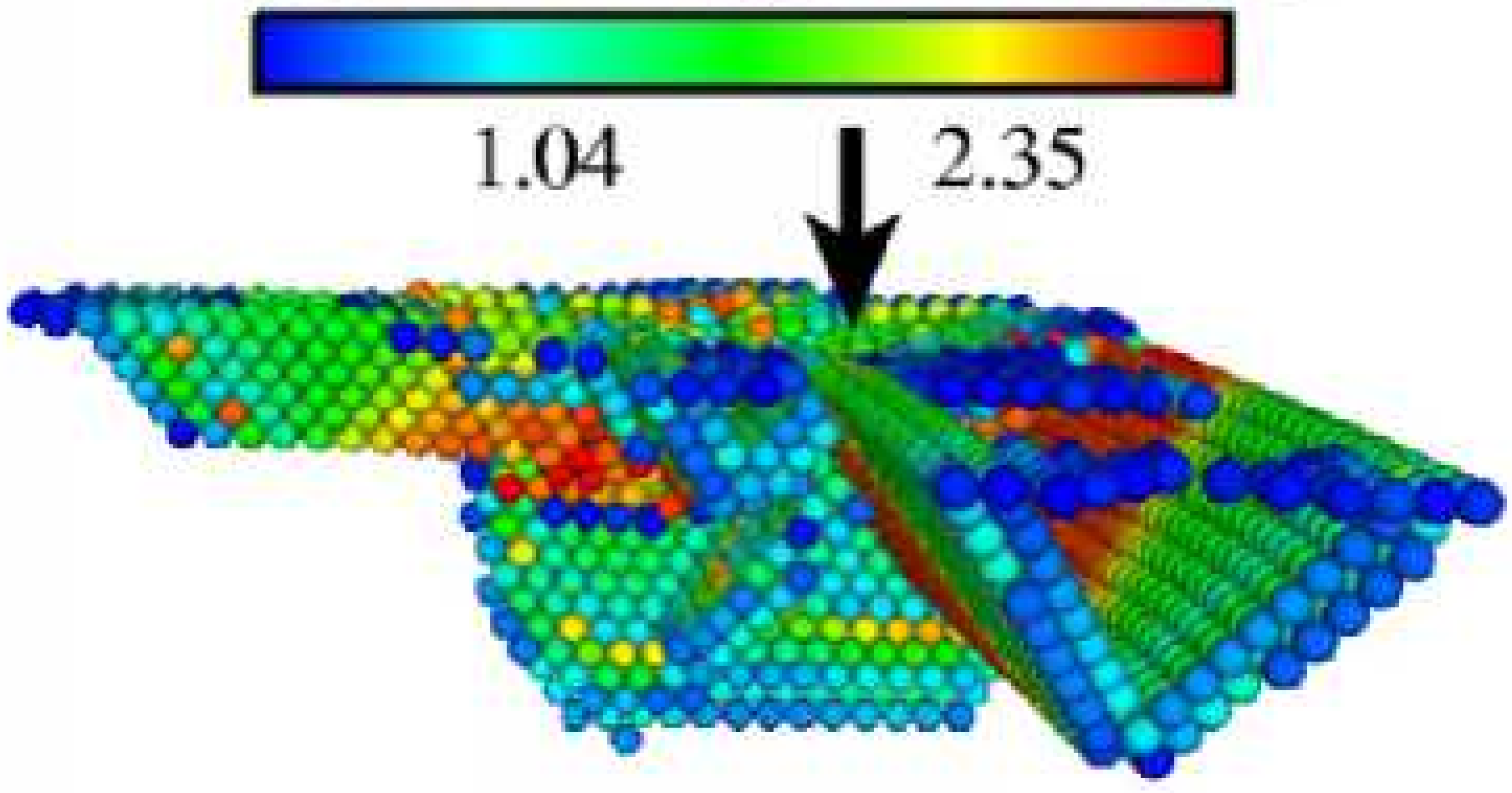